FORMATION Formation emploi

Revue française de sciences sociales

142 | Avril-Juin 2018

Génération 2010 : diversité des parcours de réussite

\title{
Emploi des jeunes sans diplôme : la prime au rural
}

Employment for young people without school-leaving certificates : the rural premium

Beschäftigung junger Menschen ohne Abschluss : Rolle der Regionpolitik

Empleo de los jóvenes sin diploma : el plus de lo rural

Joël Zaffran

\section{(2) OpenEdition}

Journals

Édition électronique

URL : http://journals.openedition.org/formationemploi/5616

DOI : 10.4000/formationemploi.5616

ISSN : 2107-0946

Éditeur

La Documentation française

Édition imprimée

Date de publication : 23 août 2018

Pagination : 99-117

ISSN : 0759-6340

Référence électronique

Joël Zaffran, «Emploi des jeunes sans diplôme : la prime au rural », Formation emploi [En ligne], 142 |

Avril-Juin 2018, mis en ligne le 23 août 2020, consulté le 30 octobre 2020. URL : http://

journals.openedition.org/formationemploi/5616; DOI : https://doi.org/10.4000/formationemploi.5616

(c) Tous droits réservés 


\title{
Emploi des jeunes sans diplôme : la prime au rural
}

\author{
Jö̈L ZAFFRAN \\ Professeur des Universités, université de Bordeaux, Faculté de sociologie, Centre Émile \\ Durkheim, Centre associé Céreq de Bordeaux
}

Résumé

\section{Emploi des jeunes sans diplôme : la prime au rural}

La situation des jeunes sans diplôme dépend de leurs caractéristiques sociales, mais aussi du tissu économique et des politiques publiques régionales en faveur de la jeunesse. Dès lors, la mesure des facteurs influençant l'accès au marché du travail ou à la formation est biaisée sans le contrôle du contexte régional. L'article met d'abord en évidence la part du niveau régional sur la situation d'emploi, de chômage ou de formation des jeunes sans diplôme. L'analyse multiniveau, menée à partir de l'enquête Génération du Céreq, estime ensuite le poids des caractéristiques sociodémographiques et scolaires sur leur situation, puis souligne l'effet du statut rural de la commune de résidence sur l'emploi. Enfin, l'article replace les résultats dans la perspective de la "régionalisation ", confiant aux Régions de nouvelles compétences en matière de formation et d'insertion des jeunes.

Mots clés : emploi des jeunes ; chômage des jeunes ; non diplômé ; abandon des études ; mesure jeune ; politique régionale de la FPC ; insertion professionnelle ; disparité régionale; région ; commune ; milieu rural ; enquête d'insertion

Abstract

\section{Employment for young people without school-leaving certificates: the rural premium}

The situation of young people without diploma depends on their social characteristics. It also depends on the economic fabric and regional public policies for youth. Hence, the measurement of factors influencing access to the labour market or access to training is biased without the control of the regional context. The article first presents the impact of the regional level on the situation of employment, unemployment or training. Then, the multilevel analysis conducted from the Generation survey from Céreq estimates the weight of the weight of sociodemographic and school characteristics on their situation, and emphasizes the effect of the rural status of the main place of residence on employment. Finally, the results are put into the perspective of "regionalization", which gives Regional Councils new competences for vocational training and employability of young people. 
Keywords: youth employment ; youth unemployment ; non graduate; drop out; youth employment scheme ; regional CVT policy ; transition from school to work ; regional disparity ; region ; municipality ; rural environment ; school-to-work transition survey.

Journal Of Economic Literarure: J 24 ; R 11

Traduction : Auteur.

La plus grande difficulté d'insertion professionnelle des jeunes sans diplôme, comparativement aux jeunes diplômés, ainsi que le poids des caractéristiques sociales sur leur situation sont des faits établis (Depp 2014) ${ }^{1}$. Souvent issus de milieux défavorisés et portés par des aspirations scolaires moins ambitieuses (Grelet \& Vivent, 2011), ils font face à des obstacles à l'accès au marché du travail plus nombreux et, quand ils y entrent, leur condition est nettement plus pénible et instable que celle des autres jeunes (Le Rhun \& al., 2013). Prenant acte de ces difficultés, des politiques publiques sont conduites à l'échelle nationale, avec un adossement aux Régions, qui contribuent, à leur manière, mais aussi selon leurs opportunités économiques et les choix opérés en matière de formation, à l'employabilité ou au retour aux études des jeunes sans diplôme (Weixler, 2014). De sorte que la mesure des caractéristiques sociales des jeunes sans diplôme sur l'emploi, la formation ou le chômage est incomplète sans un emboîtement de leur situation dans une échelle régionale, c'est-à-dire des aires urbaines et rurales administrées par un Conseil régional doté de compétences en matière d'orientation et de formation professionnelle notamment.

À la suite des travaux du Céreq (2014) ou de l'Insee (voir par exemple Bouhia \& al., 2011) sur les jeunes sans diplôme, on peut donc s'attacher à identifier d'emblée les facteurs qui accentuent ou allègent les difficultés des jeunes sans diplôme de retour en formation ou en insertion professionnelle, non sans avoir contrôlé préalablement l'effet du territoire qui intervient aux niveaux individuel et régional. Dans le premier cas, le lieu d'habitation peut représenter un handicap supplémentaire que les jeunes sans diplôme devront surmonter, comme les jeunes qui résident dans une ZUS (zone urbaine sensible), cibles des mesures liées à l'action publique mises en œuvre sur des territoires urbains précis. Dans le second cas, des différences proviennent du tissu économique de la région de résidence des jeunes (voir le rapport 2016 de l'Observatoire social des territoires de l'Insee). Ainsi, les régions du nord et du sud de la France restent les plus touchées par le chômage, avec des taux supérieurs à $10 \%$. En conséquence, les jeunes, a fortiori non diplômés, sont les plus touchés par la montée du chômage (Gaubert \& al., 2017), mais dans des contextes variables sur le plan de l'emploi (Bessone $\&$ al., 2015). Cette variation due aux territoires existe aussi dans l'accès aux

1. L'auteur remercie les membres du Département Entrées et Évolutions dans la Vie Active (DEEVA) du Céreq. L'article est une contribution à l'ANR 14-CE30-0009 Territoires et décrochages scolaires (TEDS). 
dispositifs de formation (Zaffran \& Vollet, 2018). En effet, le retour aux études dépend du nombre et de la nature des dispositifs que chaque région aura choisi de financer. En Île-de-France, par exemple, le Conseil régional rappelle, en 2016, qu'il lui incombe de renforcer le travail en commun de tous les acteurs d'un territoire, en particulier ceux qui relèvent de l'Éducation nationale et des missions locales (Rapport pour le conseil régional Île-de-France, 2016). En Région PACA, un dispositif entre la mission locale et le rectorat n'a pas eu le succès escompté par le Conseil régional. Il n'a pas survécu à la mauvaise circulation de l'information, à l'hétérogénéité des motivations et à la divergence des objectifs des intervenants (Rouaud, 2013).

Ces éléments de contexte obligent à intégrer l'effet du territoire dans l'étude de l'influence des caractéristiques sociales sur la situation des jeunes après qu'ils ont quitté l'école sans diplôme. Cet article vise donc à expliquer la situation de chômage, de retour en formation ou d'emploi des jeunes sans diplôme par des paramètres classiques (sexe, niveau de sortie, profession du père) et ad hoc (le statut urbain ou rural de la commune de résidence) à l'aide d'une modélisation multiniveau qui contrôle la variation due aux régions lors de la mesure de ces paramètres.

On s'appuie sur l'enquête Génération 2010 du Céreq (voir Encadré 1) pour, dans un premier temps, présenter la population des jeunes sans diplôme et, dans un second temps, expliquer la situation d'emploi ou de formation comparativement à la situation de chômage (voir Encadré 2).

\section{Encadré 1. Définition de l'échantillon}

Les données proviennent de l'enquête « Génération 2010 » du Céreq, qui est un dispositif d'interrogation, au printemps 2013, des 33500 sortants de tous niveaux de formation, représentatifs des 708000 jeunes sortis du système éducatif en 2010. Dans cet article, les jeunes sans diplôme ont quitté le collège, le lycée professionnel ou le lycée d'enseignement général ou bien des études secondaires générales, sans obtenir le certificat d'aptitude professionnelle (CAP), le brevet d'études professionnelles (BEP) ou le baccalauréat général, technologique ou professionnel. Ces sortants précoces ne détiennent toujours aucun titre scolaire dans les trois ans qui suivent la sortie de l'école. Les jeunes avec un diplôme sont ceux ayant obtenu, soit un CAP ou un $B E P$, soit un baccalauréat général, technologique ou professionnel après avoir quitté l'école en 2010 ou au cours des trois années suivant la sortie de l'école. Après avoir comparé les jeunes sans diplôme et les jeunes avec un diplôme, l'article réduit la focale sur les premiers.

Tous les résultats sont significatifs avec une marge d'erreur d'au moins $5 \%$. Les calculs sont effectués sur la base des effectifs pondérés. 
Préalablement à cette analyse, on s'attendait à ce que le statut urbain de la commune de résidence augmente la probabilité des jeunes sans diplôme d'être en formation ou en emploi plutôt qu'au chômage, en raison notamment des orientations de la politique de la ville et de la concentration des emplois en zone urbaine (Rapport du Commissariat général à l'égalité des territoires, 2015). Les résultats montrent l'inverse, puisque le statut rural de la commune de résidence a un effet positif sur l'emploi.

Dans un troisième temps, on met en discussion cet effet, que l'on articule ensuite à la régionalisation des politiques de formation et d'insertion des jeunes sortant du système de formation initiale sans un diplôme national ou une certification professionnelle.

\section{Qui sont les jeunes sans diplôme?}

Les jeunes sans diplôme présentent des différences attendues par rapport aux jeunes diplômés. Le Tableau 1 affiche des résultats qui vont dans le sens des données disponibles sur les sorties de l'école sans qualification (Caille, 2000 ; Afsa, 2013). Il confirme aussi le poids des facteurs scolaires et sociaux, puisque les jeunes sans diplôme sont plus souvent des garçons, des enfants d'ouvriers et d'employés, avec l'inactivité, pour situation dominante, d'un père le plus souvent né hors de France. En outre, ils sortent du système de formation sans diplôme majoritairement après un passage dans l'enseignement professionnel, avec des différences selon le niveau court (CAP ou BEP) et le niveau long (baccalauréat général, technologique ou professionnel). Enfin, ils sont plus nombreux dans les zones urbaines en général, et les zones urbaines sensibles en particulier. 
Tableau 1. Caractéristiques des jeunes sans et avec diplôme (\%)

\begin{tabular}{|c|c|c|}
\hline Caractéristiques & Jeunes sans diplôme & Jeunes avec un diplôme \\
\hline \multicolumn{3}{|l|}{ Sexe } \\
\hline Garçon & 62,5 & 49,1 \\
\hline Fille & 37,5 & 50,9 \\
\hline \multicolumn{3}{|l|}{ Profession du père } \\
\hline Ouvrier & 29,8 & 21,9 \\
\hline Employé & 21,5 & 17,8 \\
\hline Technicien, agent de maîtrise, VRP, profession intermédiaire & 5,7 & 8,6 \\
\hline Cadre, ingénieur, profession libérale, professeur & 7,1 & 24,2 \\
\hline Artisan, commerçant, chef d'entreprise & 9,7 & 10,6 \\
\hline Agriculteur & 1,6 & 3 \\
\hline \multicolumn{3}{|l|}{ Pays de naissance du père } \\
\hline En France & 75 & 82,2 \\
\hline Hors de France & 25 & 17,8 \\
\hline \multicolumn{3}{|l|}{ Niveau de sortie } \\
\hline Collège & 13,9 & \\
\hline CAP/BEP & 35,4 & \\
\hline Bac général, technologique ou professionnel & 50,7 & \\
\hline \multicolumn{3}{|l|}{ Statut de la commune de résidence } \\
\hline Urbain & 82,4 & 78,1 \\
\hline Rural & 17,1 & 21,7 \\
\hline Appartenance à une ZUS (zone urbaine sensible) & 27,8 & 14,8 \\
\hline
\end{tabular}

Lecture : parmi les jeunes sans diplôme, on compte 62,5 \% de garçons. Ils sont 49,1 \% parmi les jeunes ayant quitté l'école avec un diplôme. (voir Encadré 1 pour les catégories « jeunes sans diplômes » et « jeunes avec un diplôme »).

Source : Enquête Génération 2010 - Interrogation 2013.

Sur le plan de l'insertion professionnelle, les jeunes sans diplôme sont moins souvent en emploi que les jeunes diplômés. Le Tableau 2 montre qu'un écart de 33 \% les sépare, puisque $50 \%$ des jeunes sans diplôme sont en emploi, alors que cette situation concerne plus de $80 \%$ des jeunes diplômés. Ce résultat, trivialement prévisible, évoque le handicap de l'absence de diplôme sur le marché du travail, et rappelle que les demandes des employeurs en matière de compétences s'élèvent à mesure que les exigences de la productivité immédiate sont fortes et que diminue le plein emploi (Rose, 2012). La hausse du nombre et du niveau des diplômés qui se présentent sur le marché du travail fait de l'absence de diplôme un mauvais signal envoyé aux recruteurs (Marchal, 2015). 
Tableau 2. La situation dominante des jeunes sans et avec diplôme (\%)

\begin{tabular}{|l|c|c|c|}
\cline { 2 - 4 } \multicolumn{1}{c|}{} & Chômage & Formation & Emploi \\
\hline Sans diplôme & 38,1 & 11,7 & 50,1 \\
\hline Avec diplôme & 9 & 7,8 & 83,2 \\
\hline Total & 13,6 & 8,4 & 78 \\
\hline
\end{tabular}

Lecture: 38 \% des jeunes sans diplôme connaissent un chômage plus ou moins durable. Source : Enquête Génération 2010 - Interrogation 2013.

Les enquêtes sur les jeunes sans diplôme pointent la vulnérabilité inhérente à leur condition. Leur parcours d'insertion est chaotique, alternant des emplois précaires et des périodes plus ou moins longues d'inactivité (Mazari \& al., 2011). Les données issues de l'enquête Génération 2010 révèlent cette vulnérabilité par le nombre de mois passés au chômage, deux fois plus élevé pour les jeunes sans diplôme (14,1 mois en moyenne contre 7,4 mois), un temps d'accès au $1^{\text {er }}$ emploi presque trois fois plus long (8,6 mois contre 3,4 mois), et un nombre de mois passés en emploi plus court $(14,6$ mois contre 25,4$)$. Enfin, les jeunes sans diplôme sont plus nombreux que les autres à être en formation, ce qui est dû (comme dans l'exemple d'Île-de-France cité plus haut) à l'inflexion des mesures d'accès à la formation des jeunes sans diplôme (Aeberhardt \& al., 2011). Ces constats faits, comment les situations varient-elles à l'échelle des régions?

\section{L'effet variable du niveau régional sur la situation des jeunes sans diplôme}

La formation et l'insertion professionnelle des jeunes sans diplôme sont la combinaison, sur une aire régionale, d'une action publique avec un ensemble de sphères d'activités économiques. Des différences existent en matière de chômage, formation, emploi.

Le Graphique 1 croise le taux de chômage (en abscisse), avec le taux d'emploi (en ordonnée) et le taux de retour en formation (représenté par les cercles), répartit les régions sur une diagonale dont les pôles sont constitués par un fort taux d'inactivité (Nord-Pas-de-Calais) et un fort taux d'emploi (Auvergne). En outre, ces deux régions se distinguent sur le plan de la formation. Ainsi, le taux de retour en formation est plus élevé en Nord-Pas-de-Calais qu'en Auvergne. Au milieu du graphique, on trouve les régions qui compensent l'inactivité par le retour en formation (Provence-Alpes-Côte d'Azur ou Champagne-Ardenne), ou qui complètent leur bon taux d'emploi par un taux de retour en formation plus élevé qu'ailleurs (Aquitaine ou Bretagne). 


\section{Graphique 1. La situation dominante des jeunes sans diplôme selon l'échelle régionale}

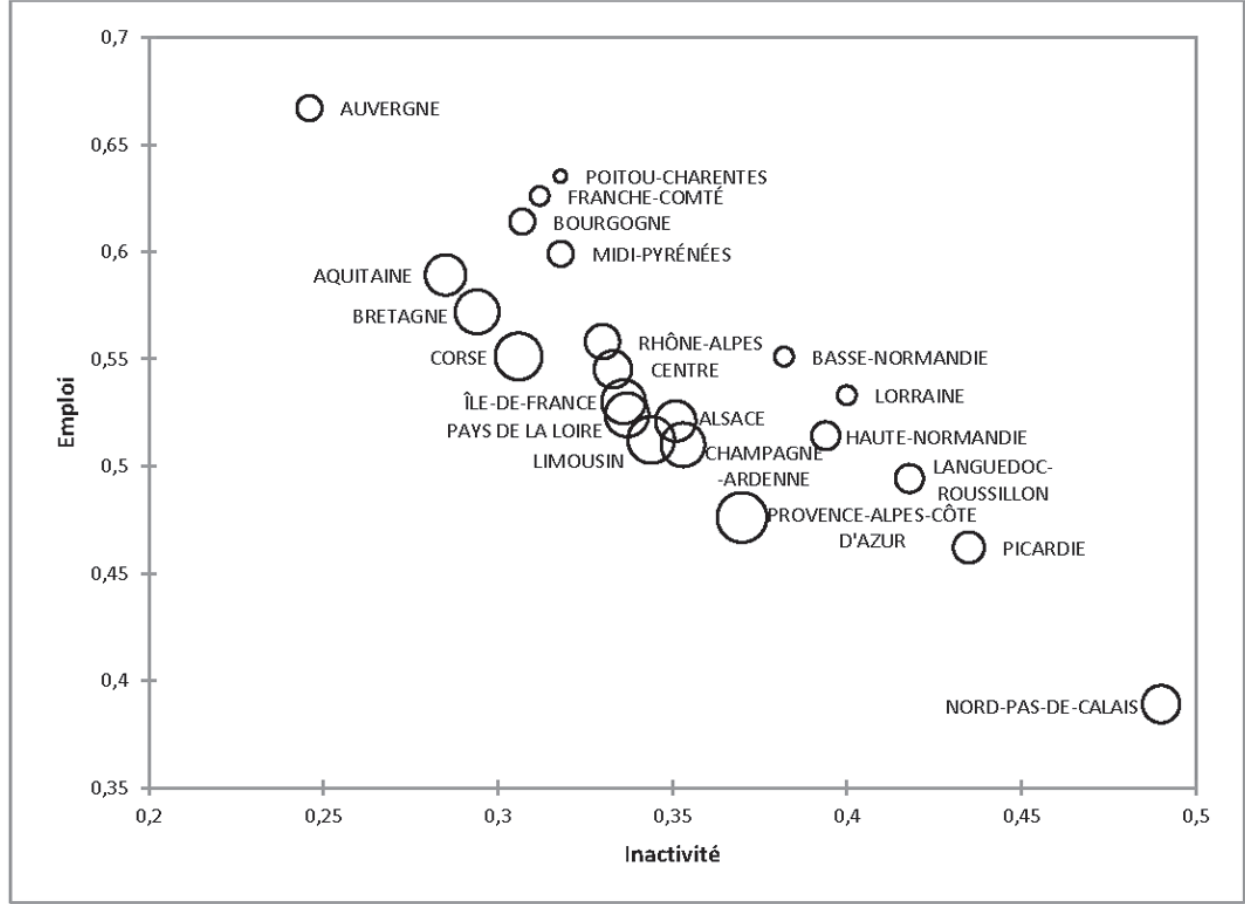

Lecture : Le retour en formation est représenté par un cercle plus ou moins large selon le taux. En Auvergne, il est égal à 11,3\%, ; en Poitou-Charentes, à 7,1 \%. La région Nord-Pas-de-Calais a un taux de chômage de 50,3 \%, un taux de retour en formation de 12.6\%, et un taux d'emploi de 37,1 \%.

Source : Enquête Génération 2010 - Interrogation 2013.

Plus avant, le Graphique 1 montre qu'il en est de la situation des jeunes sans diplôme comme du décrochage scolaire, puisque le phénomène se forme dans l'interaction entre l'individu et les dimensions de son environnement (Boudesseul, Caro, Grelet \& Vivent, 2014). Il importe donc de prendre en compte la structure hiérarchique des données pour ne pas dissocier les disparités régionales des caractéristiques sociales des jeunes et, ce faisant, éviter l'erreur écologique qui biaise l'intensité des relations analysées au niveau individuel et au niveau agrégé (Robinson, 1950).

L'intérêt de l'analyse multiniveau est de mesurer les facteurs du retour en formation, le chômage ou l'emploi des jeunes sans diplôme, en contrôlant l'effet du niveau régional².

2. La démarche est peu ou prou identique à celle que proposent Couppié, Dzikowski \& Goffette (2014, p. 28) au sujet des jeunes accueillis dans les missions locales lorraines. Ils montrent qu'à suivi et caractéristiques identiques par la mission locale, un jeune d'une communauté de communes a 1.5 fois plus de chances de connaître une insertion professionnelle qu'un jeune d'un territoire " moyen ». 
Elle estime " toutes choses égales par ailleurs " l'effet des caractéristiques individuelles sur la situation dominante d'un individu donné, à caractéristiques sociales et scolaires données et à effet du niveau régional contrôlé. De cette manière, la dépendance des observations, issue de l'imbrication des deux niveaux, n'est pas entachée des "inférences fallacieuses " de type écologique ou individualiste (cf. Encadré 2).

\section{Encadré 2. La méthode multiniveau}

La méthode multiniveau (Goldstein, 2003) mesure les liens entre des facteurs individuels et environnementaux. Surtout connue pour évaluer les effets de classe ou d'établissement scolaire sur la performance des élèves (Bressoux, 2008), elle consiste à emboîter des facteurs (les caractéristiques des élèves par exemple) au niveau 1 dans leur unité d'appartenance au niveau 2 (leur école). En articulant les données agrégées de niveau 2 et les données individuelles de niveau 1 , la méthode décompose la variance entre les deux niveaux. Elle corrige donc l'hétérogénéité des relations des élèves au sein d'une école et entre les écoles. Dans cet article, on mesure la situation des jeunes sans diplôme (formation ou emploi, comparativement à la situation de chômage) par le poids des facteurs de niveau 1 (les caractéristiques sociales) après avoir contrôlé l'effet du niveau 2 (les régions). Cette modélisation détermine jusqu'à quel point la situation des jeunes provient de leurs caractéristiques sociales (sexe, niveau de sortie), de leur capital social (1) que l'on propose d'appréhender ici par le statut (urbain ou rural) de la commune de résidence (2), et de la profession du père (3). Le Tableau 3 présente les résultats d'une analyse multiniveau multinomiale qui prend la situation dominante des jeunes sans diplôme pour variable cible, et la partage en trois modalités : chômage (C), retour en formation (F), emploi (E). II compare la probabilité d'être en formation plutôt qu'au chômage (modèles 1 et 1a), et d'être en emploi plutôt qu'au chômage (modèles 2 et 2a).

(1) Voir l'article cité de Couppié, Giret \& Moullet (2010).

(2) Le type rural ou urbain de la commune permet d'appréhender la capacité des acteurs locaux à réguler des problèmes collectifs dans un contexte donné (voir à ce sujet Pasquier, 2012 ; ou l'exemple des réseaux locaux de persévérance présenté dans la dernière partie de l'article).

(3) Le lieu de résidence en zone urbaine prioritaire de la politique de la ville est un facteur classique de diminution de la probabilité d'être en emploi plutôt qu'au chômage (Okba, 2014 ; Couppié, 2013). Or, le croisement de la variable " appartenance à une ZUS » (zone urbaine sensible) avec la situation des jeunes sans diplôme n'est pas significatif. De ce fait, nous l'avons exclue de notre modèle, tout comme le pays de naissance du père.

La dernière ligne du Tableau 3 indique que la variance à l'échelle régionale, pour une estimation à $95 \%$, entraîne une probabilité moyenne d'être en formation plutôt qu'au chômage, comprise entre 0,12 et 0,40 , quand la probabilité d'être en emploi plutôt qu'au chômage varie de 0,45 à 0,74 . 
Ces variations ont une signification pratique, puisqu'elles pointent des différences d'une région à l'autre, plus marquées dans la situation d'emploi que dans la situation de formation. Autrement dit, il existe bien des différences d'une région à l'autre dans les chances d'être en formation ou en emploi plutôt qu'au chômage. Il est évident que ces différences s'expliquent aussi par les caractéristiques des jeunes sans diplôme, que le contrôle du niveau régional permet de mesurer sans les biais du modèle logit classique.

Tableau 3. Modèle multiniveau multinomial expliquant la situation de formation ou d'emploi par rapport à la situation de chômage des jeunes sans diplôme

\begin{tabular}{|c|c|c|c|c|}
\hline Paramètres & $\begin{array}{c}\text { Modèle vide } 1 \\
\text { C( (réf.)/F }\end{array}$ & $\begin{array}{l}\text { Modèle1a } \\
\text { C (ré́f.)F }\end{array}$ & $\begin{array}{c}\text { Modèle vide } 2 \\
\text { C (réf.)/E }\end{array}$ & $\begin{array}{c}\text { Modèle 2a } \\
\text { C(réf.)/E }\end{array}$ \\
\hline \multicolumn{5}{|l|}{ Effets fixes } \\
\hline Constante & $-1,18$ & $-0,98$ & 0,41 & 0,62 \\
\hline $\begin{array}{l}\text { Sexe } \\
\text { Garçon } \\
\text { Fille (réf.) }\end{array}$ & & NS & & $0,35(1,41)$ \\
\hline $\begin{array}{l}\text { Profession du père } \\
\text { Agriculteur } \\
\text { Artisan } \\
\text { Cadre } \\
\text { Technicien } \\
\text { Employé } \\
\text { Ouvrier (réf) }\end{array}$ & & $\begin{array}{c}\text { NS } \\
0,39(1,49) \\
0,85(2,33) \\
0,49(1,63) \\
\text { NS }\end{array}$ & & $\begin{array}{c}0,81(2,26) \\
0,37(1,45) \\
0,43(1,55) \\
0,36(1,43) \\
\text { NS }\end{array}$ \\
\hline $\begin{array}{l}\text { Niveau de sortie } \\
\text { Collège } \\
\text { CAP/BEP } \\
\text { Bac (réf.) }\end{array}$ & & $\begin{array}{l}-0,51(0,61) \\
-0,59(0,55)\end{array}$ & & $\begin{array}{l}-1,61(0,21) \\
-0,62(0,54)\end{array}$ \\
\hline $\begin{array}{l}\text { Statut de la commune } \\
\text { Rurale } \\
\text { Urbain (réf) }\end{array}$ & & NS & & $0,21(1,23)$ \\
\hline Effets aléatoires & & & & \\
\hline Variance des constantes & 0,16 & 0,25 & 0,10 & 0,13 \\
\hline $\begin{array}{l}\text { Probabilité moyenne calculée à partir du } \\
\text { logarithme des odds ratio (estimation à } \\
95 \% \text { ) }\end{array}$ & {$[0,12 ; 0,40]$} & & {$[0,45 ; 0,74]$} & \\
\hline
\end{tabular}

Lecture : dans le modèle $1 b$, la probabilité toutes choses égales par ailleurs d'être en emploi plutôt qu'au chômage augmente pour un garçon par rapport à une fille. Pour celui-ci, le coefficient est de 0,35 , et le rapport de chances de 1,41. Cela signifie que la probabilité d'être en formation sur celle d'être au chômage, pour un garçon, comparé à ce même rapport de probabilité pour une fille, est 1,41 fois plus élevée. NS équivaut à un coefficient non significatif.

Source : Enquête Génération 2010 - Interrogation 2013. 
Les effets fixes du modèle 1a et du modèle 2 a se lisant comme dans une analyse de régression, on voit d'emblée que le coefficient associé au genre a un impact positif dans le modèle 2a, et aucun impact dans le modèle 1a. La probabilité d'être en emploi plutôt qu'au chômage croît avec le genre, à autres caractéristiques fixées. Le rapport de chances (odds ratio), présenté entre parenthèses à la suite du coefficient, propose une interprétation plus directe. Pour un garçon sans diplôme, la probabilité d'être en emploi sur celle d'être au chômage est de 1,41 environ fois plus élevée, comparée à ce même rapport de probabilité pour une fille sans diplôme. Ce résultat sous-entend que l'effet des facteurs personnels, familiaux et scolaires, que l'on sait plus important que les garçons (Lessard \& al., 2007), amène les filles à endosser les responsabilités liées à la famille (ou à la maternité). Leur préférence pour les rôles féminins traditionnels serait une échappatoire au défi du marché du travail qui est d'autant plus élevé que l'absence de diplôme accroît leur vulnérabilité. Autrement dit, l'inégalité des sexes entre jeunes sans diplôme se loge dans le choix des filles pour la famille, qui leur offrirait l'intégration qu'elles craignent de ne pas trouver (ou pas immédiatement ${ }^{3}$ ) par le travail (Bordigoni, 2000) $)^{4}$.

Des différences apparaissent aussi selon la profession du père ${ }^{5}$ et le niveau de sortie de l'école. Les chances de retourner en formation sont plus élevées chez les jeunes sans diplôme dont le père est cadre (2,33 fois plus élevées), que chez ceux dont le père est ouvrier. Quant au niveau de sortie scolaire des modèles 1a et $2 \mathrm{a}$, les jeunes ayant arrêté leurs études par rapport aux jeunes sortis au niveau bac (général, technologique ou professionnel), les chances d'être en formation ou en emploi plutôt qu'au chômage sont moindres pour les premiers comparativement aux seconds. L'explication, avancée supra, de la différence entre diplômés et non-diplômés, due à la nature du signal que l'absence de diplôme envoie aux employeurs, se prolonge ici, puisque l'insertion des jeunes sans diplôme dépend, pour une part, de leur niveau d'études au moment de quitter précocement l'école. En l'espèce, la sortie précoce au niveau du baccalauréat est plus favorable à l'emploi (et à la formation) qu'au niveau du collège ou d'un diplôme d'enseignement professionnel de niveau V (CAP ou BEP).

Nonobstant ces constats, notre modélisation incite à s'attarder sur un résultat, sinon inattendu, du moins peu connu, alors qu'il revêt une importance sur le plan sociologique, à savoir l'effet du statut rural de la commune de résidence des jeunes sans

3. Cette situation peut donc être transitoire, auquel cas la famille serait un « refuge » momentané.

4. Pour le vérifier, une analyse genrée approfondie de l'enquête Génération est nécessaire.

5. L'analyse menée par Aliaga \& Lê (2016) met en évidence le poids de l'origine géographique et sociale dans l'insertion des jeunes issus de l'immigration. Ces derniers rencontrent davantage de difficultés pour accéder à un emploi, et notamment à un emploi stable. À caractéristiques comparables, ils ont une probabilité plus faible d'être actifs (en emploi ou chômeurs) lorsqu'ils sont immigrés (- 14 points) ou descendants d'immigrés ( -4 points). Alors que l'on s'attendait à un effet du lieu de naissance du père sur la situation des jeunes sans diplôme, le Tableau 3 indique une absence d'effet. 
diplôme sur leur situation d'emploi ${ }^{6}$. Si cet effet n'est pas significatif dans le modèle 1a, il l'est dans le modèle $2 \mathrm{a}$. La probabilité d'être en emploi sur la probabilité d'être au chômage, pour des jeunes sans diplôme ayant les mêmes caractéristiques observables, est 1,23 fois plus élevée quand la commune de résidence est rurale ${ }^{7}$. Ce résultat fait d'abord écho aux chances plus fortes d'être en emploi quand le père est agriculteur $(2,26)$ que cadre $(1,55)$ ( $c f$. modèle $2 \mathrm{a}$ ). Il se traduit ensuite dans les différences (toutes significatives) de position des jeunes sans diplôme au moment de l'enquête. Par exemple, les jeunes ruraux sans diplôme sont plus nombreux $(67,2 \%)$ que les jeunes urbains sans diplôme (63\%) à déclarer avoir arrêté leurs études pour entrer dans la vie active. De même, les premiers sont plus nombreux (26,1 \%) que les seconds (24,4\%) à avoir trouvé un emploi après l'arrêt précoce des études. Concernant l'opinion sur le parcours et l'avenir professionnel, les jeunes ruraux sans diplôme sont plus nombreux à considérer que leur situation actuelle leur convient $(53 \%$, contre 47,3\% pour les urbains). De plus, les premiers sont $39 \%$ à déclarer avoir eu une ou plusieurs expériences professionnelles, contre $34,6 \%$ pour les seconds.

\section{La prime au rural pour l'emploi}

L'usage de focaliser l'attention sur les zones urbaines, de surcroît lorsqu'elles sont " sensibles ", conduit à négliger, voire à dénigrer, les zones rurales. Or, les résultats montrent que les jeunes ruraux sans diplôme ont une probabilité plus élevée d'être en emploi comparativement à leurs homologues urbains. Cette " prime au rural » se retrouve dans la situation des jeunes au moment de l'enquête. Ainsi, 49,1 \% des jeunes ruraux sans diplôme sont en emploi, contre 42,8 \% des jeunes urbains sans diplôme (cf. Tableau 4).

6. Une comparaison entre plusieurs territoires couverts par les missions locales entérine ce résultat (Poirier, 2015). Elle montre que les jeunes résidant dans des communes rurales sont majoritairement des actifs $(52,2 \%)$, contrairement aux jeunes des villes préfectures (42,3\%), et que le taux de chômage de ces jeunes actifs est de 4 points inférieur à celui des jeunes résidant dans les villes préfectures.

7. Pour vérifier que l'effet rural n'est pas dû aux migrations des jeunes urbains vers les bassins d'emploi en zone rurale, nous avons calculé un taux d'immobilité géographique des jeunes sans diplôme. Celui-ci est supérieur à $80 \%$, et l'indice de mobilité pour les communes rurales est de 4,2. Cette valeur élevée indique une faible fluidité géographique, puisque la sédentarité est quatre fois plus importante que dans le cas d'un nomadisme parfait. On peut donc considérer que les jeunes ruraux sont relativement immobiles, et que l'effet positif de la dimension rurale sur la situation dominante ne provient pas d'un déplacement des jeunes, d'une part, d'une aire urbaine à une aire rurale, d'autre part, d'une aire rurale à une autre aire rurale. 
Tableau 4. Situation des jeunes à la date de l'enquête, selon le statut de la commune de résidence $(\%)$

\begin{tabular}{|l|c|c|c|}
\cline { 2 - 4 } \multicolumn{1}{c|}{} & Emploi & Chômage & Formation \\
\hline Rural & 49,1 & 42,2 & 8,7 \\
\hline Urbain & 42,8 & 46,8 & 10,4 \\
\hline
\end{tabular}

Lecture : sur 100 jeunes ruraux, 49,1 sont en emploi au moment de l'enquête. Source : Enquête Génération 2010 - Interrogation 2013.

Cet effet du statut de la commune de résidence se retrouve dans trois domaines qui sont les séquences d'emploi, la situation selon le niveau et la spécialité de sortie précoce, la position professionnelle.

Dans le premier cas, le nombre moyen de séquences est un peu plus important chez les jeunes ruraux $(1,60)$ que parmi les jeunes urbains $(1,42)$. Par ailleurs, le temps d'accès au $1^{\text {er }}$ emploi en nombre de mois est plus court pour les jeunes ruraux, soit 7,10 mois en moyenne, contre 8,75 mois en moyenne pour les jeunes urbains. En revanche, les séquences de formation et de reprise d'études sont moins nombreuses chez les jeunes ruraux, puisqu'un écart de 1,7 les sépare des jeunes urbains.

Dans le deuxième cas, la sortie précoce au niveau IV du baccalauréat est plus propice à l'insertion professionnelle, en zones urbaines comme en zones rurales, qu' une formation de niveau V. En revanche, la filière au niveau IV, quelle qu'elle soit, crée une différence entre les territoires. On voit en effet que la part des jeunes sans diplôme en emploi présente toujours un écart de 8 à 14 points environ, à l'avantage des jeunes ruraux (cf. tableau 5)8 .

Tableau 5. Situation des jeunes selon le statut de la commune de résidence et le niveau et la spécialité de sortie (\%)

\begin{tabular}{|l|l|c|c|c|}
\cline { 2 - 5 } \multicolumn{1}{c|}{} & \multicolumn{1}{c|}{ Niveau et filières scolaires } & Chômage & Formation & Emploi \\
\hline \multirow{4}{*}{ Urbain } & Collège & 62,7 & 14,8 & 22,5 \\
& Cap-Bep & 47,3 & 10,2 & 42,5 \\
& Bac Pro & 36,2 & 8,7 & 55,2 \\
& Bac Techno & 30,6 & 16,0 & 53,4 \\
& Bac Général & 26,0 & 21,6 & 52,4 \\
\hline \multirow{5}{*}{ Rural } & Collège & 59,9 & 13,0 & 27,1 \\
& Cap-Bep & 46,8 & 8,5 & 44,8 \\
& BacPro & 25,3 & 5,6 & 69,1 \\
& Bac Techno & 18,8 & 15,2 & 66,1 \\
& Bac Général & 31,2 & 9,3 & 59,6 \\
\hline
\end{tabular}

Lecture : $22,5 \%$ de jeunes urbains ayant quitté l'école au niveau du collège sont en emploi. Le taux passe à $27,1 \%$ chez les jeunes ruraux. Source : Enquête Génération 2010 - Interrogation 2013.

8. Le tableau montre aussi que le territoire urbain est plus propice aux situations de formation, en particulier lorsque la sortie précoce a eu lieu en baccalauréat général. 
Dans le troisième cas, la position professionnelle à l'embauche déclarée à la première séquence d'emploi, selon le territoire, marque des différences significatives entre les jeunes (Tableau 6). Si la part des «employés" est la plus importante parmi tous les jeunes sans diplôme, les emplois de " manœuvre ou ouvrier spécialisé » et " ouvrier qualifié ou travailleur agricole " sont surreprésentés chez les jeunes ruraux, quand les autres emplois le sont chez les jeunes urbains.

Tableau 6. Position professionnelle à l'embauche déclarée et statut de la commune $1^{\text {re }}$ séquence d'emploi en $\%$

\begin{tabular}{|l|c|c|c|}
\cline { 2 - 4 } \multicolumn{1}{c|}{} & Rural & Urbain & \% sur total \\
\hline Manœuvre ou ouvrier spécialisé & 18,5 & 14,8 & 14,8 \\
\hline Ouvrier qualifié ou travailleur agricole & 11,6 & 10,3 & 11,3 \\
\hline Agent de maîtrise & 1,6 & 1,8 & 1,5 \\
\hline Technicien dessinateur VRP & 3,1 & 3,5 & 3,9 \\
\hline Employé & 52 & 55 & 54,4 \\
\hline Employé qualifié & 13 & 14,4 & 13,8 \\
\hline Personnels catégorie Cou D fonction publique & 0,3 & 0,1 & 0,2 \\
\hline
\end{tabular}

Lecture : sur 100 jeunes ruraux sans diplôme en emploi, 18,5 occupent un poste de manœuvre ou d'ouvrier spécialisé.

Source : Enquête Génération 2010 - Interrogation 2013.

On fait l'hypothèse que tous ces écarts sont dus à la convergence de l'offre locale d'emplois disponibles avec les attentes des employeurs ruraux, moins élevées que celles des employeurs en zone urbaine. Alors que l'emploi dans les aires urbaines dépend du niveau de diplôme obtenu, qui devra être jugé suffisant pour participer à la dynamique de l'innovation (CERC, 2008), l'absence de diplôme en zone rurale ne constitue pas un handicap identique sur le marché du travail. Toutes proportions gardées, et en contrôlant les caractéristiques du tissu économique local, les employeurs en zone urbaine auraient des exigences que n'auraient pas les employeurs ruraux. Le désavantage de l'absence de diplôme sur l'insertion professionnelle serait donc allégé en zones rurales, qui sont moins tenues par les enjeux économiques urbains de la création. Ainsi, à quelque chose malheur est bon, puisque bien que faiblement dotés en facteurs classiques de l'innovation, des territoires ruraux améliorent l'insertion des jeunes sans diplôme. Par conséquent, cela interroge le caractère critiquable de la priorité accordée aux territoires urbains, en particulier aux ZUS. Le rapport du Commissariat général à l'égalité des territoires, de 2015, pointe l'écart entre les moyens alloués à la politique publique de la ville (qu'il s'agisse, soit de l'aide à l'implantation et du soutien d'activités, soit d'un accès prioritaire aux dispositifs d'insertion ou à l'emploi) et les résultats obtenus, quand le rapport annuel de l'Observatoire national de la politique de la ville souligne que les mesures peinent à réduire les obstacles d'accès au marché du travail, plus importants en ZUS (Dieusaert, 2016). 
Outre la convergence de l'offre locale d'emplois disponibles avec les attentes des employeurs ruraux, une seconde hypothèse, complémentaire à la première, viendrait souligner l'importance du réseau d'interconnaissance et de la mobilisation des acteurs territoriaux. Car s'il est évident que l'insertion professionnelle des jeunes sans diplôme dépend, pour une grande part, du tissu économique local, l'effet du statut de la commune de résidence s'expliquerait par la capacité des acteurs locaux à réguler des problèmes collectifs sur un territoire donné.

Dès lors, quid des moyens dont disposent ces territoires pour agir sur l'accès à un emploi, dût-il être pénible ou peu rémunérateur ? Si l'enquête Génération ne permet pas d'y répondre directement, l'évaluation de l'expérimentation des "Réseaux locaux aquitains pour la persévérance " met au jour des différences dans les modes d'accompagnement des jeunes sans diplôme (Plessard \& Zaffran, 2012). Lors de cette évaluation, trois réseaux implantés sur trois territoires d'Aquitaine ont fait l'objet d'une évaluation : en Haute Gironde, sur les Hauts-de-Garonne et dans le Marmandais. Pour chacun, l'objectif était de prévenir les ruptures scolaires, d'améliorer la prise en charge des jeunes sans diplôme, et de traiter leurs difficultés d'accès à une qualification ou un emploi. Le travail partenarial et la collaboration des structures d'accompagnement des jeunes sont apparus comme les ingrédients de l'interconnaissance locale. Dans le premier territoire, une aire à dominante rurale, les interventions auprès des jeunes sont concertées. De plus, le réseau est structuré par des liens forts de tous les acteurs de l'éducation et de l'insertion avec les élus locaux. Dans les autres territoires, le réseau est moins structuré, mais c'est dans les Hauts-deGaronne, un territoire fortement urbanisé, que le travail des acteurs est contrarié par des enjeux étrangers à l'insertion des jeunes. Le réseau local d'acteurs en charge de l'insertion des jeunes souffre d'une proximité géographique avec le Conseil régional et d'une absence de lisibilité des dispositifs en faveur des jeunes sans diplôme.

L'exemple des « Réseaux locaux aquitains pour la persévérance » confirme les observations de Lambert \& Roudet (1995), à savoir que les jeunes ruraux bénéficient des liens plus forts et durables avec leur territoire que les jeunes urbains. Il fait apparaître les groupes d'acteurs ruraux comme des entités liées par des relations, formelles et informelles, dont la force est un préalable à la mobilisation effective autour du problème de l'emploi. Mais si les jeunes ruraux bénéficient d'une interconnaissance fonctionnelle plus riche que les jeunes urbains, cette ressource est inégalement distribuée sur les territoires ruraux. Le cas du Marmandais révèle qu'elle prend une forme différente selon que la commune est incluse dans un territoire rural dynamique ou une aire sinistrée, selon l'éloignement aux ressources économiques, scolaires et culturelles, selon la proximité avec le pouvoir central, et enfin selon la capacité des acteurs locaux à s'organiser et se coordonner pour faire face à un problème collectif.

Autrement dit, l'effet de la commune de résidence ne permet pas de rassembler, sous un même label, des territoires ruraux que l'on sait peu comparables sur le plan sociologique (Sencébé, 2011 ; Morin, 2011). Le monde rural, souvent associé à l'autochtonie et à la 
succession des générations dans un même lieu (Rétière, 1994 ; Renahy, 2005), n’est pas homogène. En conséquence, "l'effet du rural " ne doit pas gommer les différences qui le renforcent ou l'affaiblissent, soit les différences entre les garçons et les filles (Arrighi, 2004), entre les territoires par nature différents sur le plan spatial - il n'y a pas en effet une campagne, mais des campagnes - entre les jeunesses - il n'y a pas non plus une jeunesse rurale, mais des jeunesses rurales (Lemêtre \& Orange, 2016). Prendre acte de cette hétérogénéité est un préalable à l'observation raisonnée des ressources locales dont bénéficieraient les jeunes ruraux, et dont seraient privés les jeunes urbains.

\section{Conclusion}

Après le transfert, en 1993, de la compétence en matière de formation professionnelle aux Conseils régionaux, de nouvelles compétences leur sont confiées dans le cadre de la loi du 7 août 2015 portant sur la nouvelle organisation territoriale de la République. S'agissant des sorties précoces du système scolaire, la convention signée par l'Association des Régions de France (ARF), le ministre de l'Éducation nationale et le ministre du Travail attribue aux Régions la responsabilité d'animer la lutte contre le décrochage scolaire et pour l'insertion des jeunes sans diplôme.

Or, l'absence de diplôme n'explique pas tout, puisque nos résultats montrent, d'une part, que des inégalités, de nature différente, existent entre les jeunes eux-mêmes ; d'autre part, que certaines caractéristiques sociales accroissent les difficultés, quand la nature des territoires les diminue. C'est l'originalité de nos résultats de montrer qu'en matière d'insertion professionnelle des jeunes sans diplôme, la campagne fait mieux que la ville.

Si les territoires ruraux pâtissent objectivement d'une faible dotation en facteurs classiques de l'innovation - en l'occurrence la concentration de talents et d'individus de la classe créative, la recherche et le développement, les réseaux de transport et de communication, l'accès au financement de l'innovation et au foncier à vocation économique (OCDE, 2010) - l'image disqualifiante et misérabiliste du monde rural résiste mal à nos données. En pointant l'effet du statut rural de la commune de résidence sur l'emploi des jeunes sans diplôme, nos résultats suggèrent que les jeunes ruraux profitent de dotations en capital social dont seraient privés les jeunes urbains. Sous certaines conditions - qu'il s'agirait de saisir -, et à niveau de sortie scolaire identique, les communes rurales seraient l'inverse des ZUS. Ici, la faiblesse des dotations des jeunes des ZUS empêche l'accès au marché du travail. Là, le réseau d'interconnaissance est profitable à leur insertion professionnelle.

La régionalisation est vue classiquement comme une arène politique centrale de niveau intermédiaire répondant aux besoins de la territorialisation des politiques de formation et d'emploi. Mais elle est aussi la somme d'interactions entre une variété d'acteurs dans des arènes centrales ou périphériques et aussi des aires éloignées qui creusent ou compensent les déséquilibres territoriaux. Les politiques régionales en faveur des jeunes sans diplôme sont tributaires des disparités qui singularisent les territoires des Régions, "grandes » de 
surcroît ${ }^{9}$. En cela, l'article ouvre des pistes sur la nature des configurations territoriales, et les bénéfices secondaires que les jeunes en retirent. Le préalable à leur exploration est, d'une part, de ne pas réenchanter les campagnes et de prendre la mesure de leurs recompositions actuelles ; d'autre part, de ne pas méconnaître la structuration des marchés du travail locaux et la qualité des emplois offerts. Ceci fait, elles étayeront, le cas échéant, ce résultat principal qu'en matière de sortie précoce de l'école et d'emploi, il vaut mieux être un jeune à la campagne plutôt qu'un jeune à la ville.

\section{Bibliographie}

Aeberhardt R., Crusson L., Pommier P. (2011), " Les politiques d'accès à l'emploi en faveur des jeunes : qualifier et accompagner », France, portrait social, édition 2011, Insee Références, pp. 53-172.

Afsa C. (2013), "Qui décroche?", Éducation \& formations, 84, pp 9-20.

Aliaga C., Lê J. (2016), "L'insertion des jeunes sur le marché du travail : l'emploi est majoritaire chez les plus diplômés, l'inactivité domine chez les non-diplômés ", Insee Références, pp. 43-57.

Arrighi J.-J. (2004), « Les jeunes dans l'espace rural : une entrée précoce sur le marché du travail ou une migration probable ", Formation Emploi, 87, juillet-septembre, pp. 63-78.

Bessone A. J., Dorothée O., Robin M., Vugdalic S. (2015), « Emploi et chômage dans les nouvelles régions depuis la crise ", Insee Focus, n 40, novembre.

Bordigoni M. (2000), Jeunes sans diplôme et risques d'exclusion sociale : exploitations spécifiques de l'enquête "Génération 92 " pour l'Observatoire de la Pauvreté et de l'Exclusion, Rapport final.

Boudesseul G., Caro P., Grelet Y., Vivent C. (2014), «Une analyse territoriale de l'échec scolaire ", Géographie de l'école, DEPP, n 11, pp. 99-111.

Bouhia R., Garrouste M., Lebrère A., Ricroh L., De Saint Paul T. (2011), "Être sans diplôme aujourd'hui en France : quelles caractéristiques, quels parcours et quel destin ?", Économie et Statistique, n 443 , Insee, pp. 29-50.

Bressoux P. (2008), Modélisation statistique appliquée aux sciences sociales, Bruxelles, De Boeck.

9. La limite de l'article est de ne rien dire des manières dont les jeunes sans diplôme définissent leur situation de chômage, de formation ou d'emploi, ni de leurs conditions réelles de travail, à n'en pas douter différentes selon le type d'emploi et le territoire. Sur ce plan, nous ne sommes pas en mesure de distinguer les campagnes isolées des territoires proches et dépendants des aires d'influence des grandes villes ou ayant subi des délocalisations industrielles (Coquard, 2015). 
Caille J.-P. (2000), "Qui sort sans qualification du système éducatif ? ", Éducation et Formations, 57, pp. 19-37.

CERC (2008), Un devoir national. Linsertion des jeunes sans diplôme, Conseil de l'emploi, des revenus et de la cohésion sociale, Rapport $\mathrm{n}^{\circ} 9$.

CÉREQ (2014), Quand l'école est finie ... Premiers pas dans la vie active de la génération 2010, enquête 2013.

Coquard B. (2015), Que sait-on des jeunes ruraux? Revue de littérature, INJEPR, rapport 2015/03.

Couppié T., Giret J.-F., Moullet S. (2010), «Lieu de résidence et discrimination salariale : le cas des jeunes habitant dans une zone urbaine sensible ", Économie et Statistique, $\mathrm{n}^{\circ}$ 433-434, pp. 447-70.

Couppié T. (2013), "Insertion des jeunes issus de quartiers sensibles : les hommes doublement pénalisés ", Céreq-Bref, n 309, avril.

Couppié T., Dzikowski C., Goffette C. (2014), Jeunes accueillis dans les missions locales lorraines entre 2005 et (2012) : Trajectoires-types et contextes territoriaux, Rapport CÉREQ-GIP Lorraine Parcours métiers.

Dardier A., Laïb N., Robert-Bobée I. (2013), « Les décrocheurs du système éducatif : de qui parle-t-on?", in France, portrait social, Insee Références, pp. 10-22.

DEPP (2014), "Les parcours scolaires et les résultats ", in Géographie de l'École 2014, pp. 66-85.

Dieusaert P. (2016), Inactivité et halo autour du chômage dans les quartiers prioritaires, Rapport annuel de l'Observatoire national de la politique de la ville.

Gaubert E., Henrard V., Robert A., Rouaud P. (2017), « Pas d'amélioration de l'insertion professionnelle pour les non-diplômés, Cereq-Bref, $\mathrm{n}^{\circ} 356$.

Grelet Y., Vivent C. (2011), "La course d'orientation des jeunes ruraux ", Cereq-Bref, $\mathrm{n}^{\circ} 292$, septembre.

Lambert Y., Roudet B. (1995), "Qui sont les jeunes ruraux?", Agora débats/jeunesses, n 1, pp. 47-56.

Lemêtre C., Orange S. (2016), "Jeunesses rurales - jeunesses ordinaires ", Savoir/Agir, 2016/3, n³7, pp. 9-13. DOI 10.3917/sava.037.0009.

Le Rhun B., Martinelli D., Minni C., Recotillet I. (2013), "Origine et insertion des jeunes sans diplôme ", Formations et emploi. Insee Références, pp. 23-32.

Lessard A., Fortin L., Joly J., Royer E., Marcotte D., Potvin P. (2007), « Cheminement de décrocheurs et de décrocheuses ", Revue des sciences de l'éducation, vol. 33, $\mathrm{n}^{\circ} 3$, pp. 647-662. 
Marchal E. (2015), Les embarras des recruteurs. Enquête sur le marché du travail, Paris, EHESS.

Mazari Z., Meyer V., Rouaud P., Ryk F., Winnicki P. (2011), " Le diplôme : un atout gagnant pour les jeunes face à la crise ", Cereq-Bref, 283, mars.

Morin B. (2011), "Qui habite en milieu rural ? ", Informations sociales, 2011/2, n 164, pp. 11-22.

OCDE (2010), La Stratégie de l'OCDE pour l'innovation : pour prendre une longueur d'avance, document téléchargeable.

Okba M. (2014), " Jeunes immigrés et jeunes descendants d'immigrés. Une première insertion sur le marché du travail plus difficile, en particulier pour ceux qui résident en Zus ", Dares analyses, n 074, septembre.

Pasquier R. (2012), Le pouvoir régional. Mobilisations, décentralisation et gouvernance en France, Paris, Presses de Sciences Po.

Plessard C. \& Zaffran J. (2012), Des réseaux locaux pour la persévérance et la réussite des jeunes Aquitains, Rapport d'évaluation pour le FEJ.

Poirier E., (2015), « Regards des missions locales sur la jeunesse de leurs territoires ", Pour, 2015/1, n²25, pp. 83-90.

Rapport du Commissariat général à l'égalité des territoires, (2015), Innovation et territoires de faible densité.

Rapport pour le conseil régional Île-de-France (2016), Lutte contre le décrochage scolaire, rapport CR 82-16.

Renahy N. (2005), Les gars du coin : enquête sur une jeunesse rurale, Paris, La Découverte.

Rétière J.-N. (1994), Identités ouvrières. Histoire d'un fief ouvrier en Bretagne, 1909-1990, Paris, L'Harmattan.

Robinson W. S. (1950), "Ecological Correlations and the Behavior of Individuals", American Sociological Review, vol. 15, pp. 351-357.

Rouaud P. (2013), "Évaluer un dispositif de lutte contre la rupture scolaire ", Céreq-Bref, $\mathrm{n}^{\circ} 307$, mars.

Rose J. (2012), Qu'est-ce que l'emploi non qualifié ?, Paris, La Dispute.

Sencébé Y. (2011), "Multi(ples)appartenances en milieu rural ", Informations sociales, $\mathrm{n}^{\circ} 164$, pp. 36-42.

Trancart D., Testenoire A. (2003), "Emploi non qualifié et trajectoires féminines ", $10^{\text {e }}$ Journées d'études Céreq - Lasmas-IdL, Les données longitudinales dans l'analyse du 
marché du travail, Caen, 21-23 mai 2003. Document téléchargeable : http://www. cereq.fr/gsenew/concours2008/cereq/Colloques/journees/36_Trancart.pdf

Weixler F. (2014), Évaluation partenariale de la politique de lutte contre le décrochage scolaire, rapport du SGMAP et de la DGESCO.

Zaffran J., Vollet J. (2018), Zadig après l'école. Pourquoi les décrocheurs scolaires raccrochentils ?, Lormont, Bord de L'Eau. 